Korean J. Math. 20 (2012), No. 3, pp. 293-305

\title{
MODAL, NECESSITY, SUFFICIENCY AND CO-SUFFICIENCY OPERATORS
}

\author{
YONG CHAN KIM
}

\begin{abstract}
We investigate the properties of modal, necessity, sufficiency and co-sufficiency operators. We show that their operations induce various relations, respectively.
\end{abstract}

\section{Introduction}

Pawlak [5] introduced rough set theory to generalize the classical set theory. Rough approximations are defined by a partition of the universe which is corresponding to the equivalence relation about information. An information consists of $(X, A)$ where $X$ is a set of objects and $A$ is a set of attributes, a map $a: X \rightarrow P\left(A_{a}\right)$ where $A_{a}$ is the value set of the attribute $a$. Recently, intensional modal-like logics with the propositional operators induced by relations are important mathematical tools for data analysis and knowledge processing [1-3, 6-9].

In this paper, we investigate the properties of modal, necessity, sufficiency and co-sufficiency operators. We show that their operations induce various relations, respectively.

\section{Preliminaries}

Definition 2.1. [2] Let $P(X), P(Y)$ be the families of subsets on $X$ and $Y$, respectively. Then a map $F: P(X) \rightarrow P(Y)$ is called

(1) modal operator if $F\left(\bigcup_{i \in \Gamma} A_{i}\right)=\bigcup_{i \in \Gamma} F\left(A_{i}\right), F(\emptyset)=\emptyset$,

(2) necessity operator if $F\left(\bigcap_{i \in \Gamma} A_{i}\right)=\bigcap_{i \in \Gamma} F\left(A_{i}\right), F(X)=Y$,

(3) sufficiency operator if $F\left(\bigcup_{i \in \Gamma} A_{i}\right)=\bigcap_{i \in \Gamma} F\left(A_{i}\right), F(\emptyset)=Y$,

(4) co-sufficiency operator if $F\left(\bigcap_{i \in \Gamma} A_{i}\right)=\bigcup_{i \in \Gamma} F\left(A_{i}\right), F(X)=\emptyset$.

Received July 2, 2012. Revised September 10, 2012. Accepted September 12, 2012 .

2010 Mathematics Subject Classification: 06A06, 06A15, 06B30, 54F05, 68U35.

Key words and phrases: Modal, necessity, sufficiency and co-sufficiency operators. 
(5) a dual operator $F^{\partial}$ is defined by $F^{\partial}(A)=F\left(A^{c}\right)^{c}$. Moreover, we define $F^{c}(A)=(F(A))^{c}$ and $F^{*}(A)=F\left(A^{c}\right)$.

Definition 2.2. [2,4] Let $R \subset P(X \times Y)$ be a relation. For each $A \in P(X)$, we define operations $(y, x) \in R^{-1}$ iff $(x, y) \in R$ and $[R],[[R]],\langle R\rangle,\langle\langle R\rangle\rangle,[R]^{*},\langle R\rangle^{*}: P(X) \rightarrow P(Y)$ as follows:

$$
\begin{gathered}
{[R](A)=\{y \in Y \mid(\forall x)((x, y) \in R \rightarrow x \in A)\},} \\
{[[R]](A)=\{y \in Y \mid(\forall x \in X)(x \in A \rightarrow(x, y) \in R)\}} \\
\langle R\rangle(A)=\{y \in Y \mid(\exists x \in X)((x, y) \in R, x \in A)\} \\
\langle\langle R\rangle\rangle(A)=\left\{y \in Y \mid(\exists x \in X)\left((x, y) \in R^{c}, x \in A^{c}\right)\right\}, \\
{[R]^{*}(A)=\left\{y \in Y \mid(\forall x \in X)\left((x, y) \in R \rightarrow x \in A^{c}\right)\right\}} \\
\langle R\rangle^{*}(A)=\left\{y \in Y \mid(\exists x \in X)\left((x, y) \in R, x \in A^{c}\right)\right\} .
\end{gathered}
$$

TheOREM 2.3. [2] Let $R \subset P(X \times Y)$ be a relation.

(1) $\langle R\rangle$ is a modal operator and $[R]$ is a necessity operator with $\langle R\rangle(A)=\left([R]\left(A^{c}\right)\right)^{c}=[R]^{\partial}(A)$, for each $A \in P(X)$.

(2) If $F: P(X) \rightarrow P(Y)$ is a modal operator on $P(X)$, there exists a unique relation $R_{F} \subset P(X \times Y)$ such that $\left\langle R_{F}\right\rangle=F$ and $\left[R_{F}\right]=F^{\partial}$ where $(x, y) \in R_{F}$ iff $y \in F(\{x\})$.

(3) $R_{\langle R\rangle}=R$.

3. Modal, necessity, sufficiency and co-sufficiency operators

Lemma 3.1. Let $F, G: P(X) \rightarrow P(Y)$ be operators. Then the following properties hold:

(1) $\left(F^{\partial}\right)^{\partial}=F,\left(F^{c}\right)^{c}=F$ and $\left(F^{*}\right)^{*}=F$.

(2) $\left(F^{\partial}\right)^{*}=\left(F^{*}\right)^{\partial},\left(F^{\partial}\right)^{c}=\left(F^{c}\right)^{\partial}$ and $\left(F^{*}\right)^{c}=\left(F^{c}\right)^{*}=F^{\partial}$.

(3) $(F \cup G)^{\partial}=F^{\partial} \cap G^{\partial},(F \cup G)^{*}=F^{*} \cup G^{*}$ and $(F \cup G)^{c}=F^{c} \cap G^{c}$.

(4) $F, G: P(X) \rightarrow P(Y)$ are modal operators, then $F \cup G$ is a model operator and it's dual operator $F^{\partial} \cap G^{\partial}$ is a necessity operator.

(5) $F, G: P(X) \rightarrow P(Y)$ are necessity operators, then $F \cap G$ is a necessity operator and it's dual operator $F^{\partial} \cap G^{\partial}$ is a model operator. 
Proof. (1) $\left(F^{\partial}\right)^{\partial}(A)=\left(F^{\partial}\left(A^{c}\right)\right)^{c}=F(A)$.

(2) $\left(F^{\partial}\right)^{*}(A)=F^{\partial}\left(A^{c}\right)=F^{c}(A)=\left(F^{*}\left(A^{c}\right)\right)^{c}=\left(F^{*}\right)^{\partial}(A)$.

$\left(F^{\partial}\right)^{c}(A)=\left(F^{\partial}(A)\right)^{c}=F\left(A^{c}\right)=\left(F^{c}\left(A^{c}\right)\right)^{c}=\left(F^{c}\right)^{\partial}(A)$.

(3) $(F \cup G)^{\partial}(A)=\left((F \cup G)\left(A^{c}\right)\right)^{c}=\left(F\left(A^{c}\right)\right)^{c} \cap\left(F\left(A^{c}\right)\right)^{c}=F^{\partial}(A) \cap$ $G^{\partial}(A)$. Other cases are similarly proved.

(4) and (5) are easily proved from (3).

Lemma 3.2. (1) A map $F: P(X) \rightarrow P(Y)$ is a modal operator iff $F^{\partial}: P(X) \rightarrow P(Y)$ is a necessity operator.

(2) A map $F: P(X) \rightarrow P(Y)$ is a sufficiency operator iff $F^{\partial}$ : $P(X) \rightarrow P(Y)$ is a co-sufficiency operator operator.

(3) A map $F: P(X) \rightarrow P(Y)$ is a modal operator iff $F^{c}: P(X) \rightarrow$ $P(Y)$ is a sufficient operator.

(4) A map $F: P(X) \rightarrow P(Y)$ is a co-sufficiency operator iff $F^{c}$ : $P(X) \rightarrow P(Y)$ is a necessity operator operator.

(5) A map $F: P(X) \rightarrow P(Y)$ is a sufficiency operator iff $F^{*}$ : $P(X) \rightarrow P(Y)$ is a necessity operator operator.

(6) A map $F: P(X) \rightarrow P(Y)$ is a modal operator iff $F^{*}: P(X) \rightarrow$ $P(Y)$ is a co-sufficiency operator.

Proof. (1) Let $F: P(X) \rightarrow P(Y)$ be a modal operator.

$$
\begin{aligned}
F^{\partial}\left(\bigcap_{i \in \Gamma} A_{i}\right) & =\left(F\left(\bigcup_{i \in \Gamma} A_{i}^{c}\right)\right)^{c}=\left(\bigcup_{i \in \Gamma} F\left(A_{i}^{c}\right)\right)^{c} \\
& =\bigcap_{i \in \Gamma}\left(F\left(A_{i}^{c}\right)\right)^{c}=\bigcap_{i \in \Gamma} F^{\partial}\left(A_{i}\right) . \\
F^{\partial}(X) & =\left(F\left(X^{c}\right)\right)^{c}=(F(\emptyset))^{c}=Y .
\end{aligned}
$$

Conversely, $\left(F^{\partial}\right)^{\partial}(A)=\left(F^{\partial}\left(A^{c}\right)\right)^{c}=F(A)$.

$$
\begin{aligned}
F\left(\bigcup_{i \in \Gamma} A_{i}\right) & =\left(F^{\partial}\left(\bigcap_{i \in \Gamma} A_{i}^{c}\right)\right)^{c}=\left(\bigcap_{i \in \Gamma} F^{\partial}\left(A_{i}^{c}\right)\right)^{c} \\
& =\bigcup_{i \in \Gamma}\left(F^{\partial}\left(A_{i}^{c}\right)\right)^{c}=\bigcup_{i \in \Gamma} F\left(A_{i}\right) . \\
F(\emptyset) & =\left(F^{\partial}\left((\emptyset)^{c}\right)\right)^{c}=F(X)^{c}=\emptyset .
\end{aligned}
$$


(2), (3) and (4) are similarly proved as same in (1).

TheOREM 3.3. Let $R \subset P(X \times Y)$ be a relation.

(1) $\langle\langle R\rangle\rangle^{*}$ is a modal operator and $[[R]]^{*}$ is a necessity operator with $\langle\langle R\rangle\rangle^{*}(A)=\left([[R]]^{*}\left(A^{c}\right)\right)^{c}=\left([[R]]^{*}\right)^{\partial}(A)$ for each $A \in P(X)$.

(2) If $F: P(X) \rightarrow P(Y)$ is a modal operator on $P(X)$, there exists a unique relation $R_{F} \subset P(X \times Y)$ such that $\left\langle\left\langle R_{F}\right\rangle\right\rangle^{*}=F$ and $\left[\left[R_{F}\right]\right]^{*}=$ $F^{\partial}$ where $(x, y) \in R_{F}$ iff $y \in F(\{x\})^{c}$.

(3) $R_{\langle\langle R\rangle\rangle^{*}}=R$.

Proof. (1) We have $\langle\langle R\rangle\rangle^{*}(A)=\left([[R]]^{*}\left(A^{c}\right)\right)^{c}=\left([[R]]^{*}\right)^{\partial}(A)$ from:

$$
\begin{aligned}
y \in\left([[R]]^{*}\left(A^{c}\right)\right)^{c} & \text { iff }((\forall x \in X)(X \in A \rightarrow(x, y) \in R))^{c} \\
& \text { iff }\left((\forall x \in X)\left((x, y) \in R^{c}, x \in A\right)^{c}\right)^{c} \\
& \text { iff }(\exists x \in X)\left((x, y) \in R^{c}, x \in A\right) \\
& \text { iff } y \in\langle\langle R\rangle\rangle^{*}(A) .
\end{aligned}
$$

(2) Since $A=\bigcup_{x \in A}\{x\}$ and $F(A)=\bigcup_{x \in A} F(\{x\})$, we have

$$
\begin{aligned}
y \in\left\langle\left\langle R_{F}\right\rangle\right\rangle^{*}(A) & \text { iff }(\exists x \in X)\left((x, y) \in R_{F}^{c} \& x \in A\right) \\
& \text { iff }(\exists x \in X)(y \in F(\{x\}) \& x \in A) \\
& \text { iff } y \in \bigcup_{x \in A} F(\{x\})=F\left(\bigcup_{x \in A}\{x\}\right)=F(A) . \\
y \in\left[\left[R_{F}\right]\right]^{*}(A) & \text { iff } \left.(\forall x \in X)\left(x \in A^{c} \rightarrow(x, y) \in R_{F}\right)\right) \\
& \text { iff } \left.(\forall x \in X)\left((x, y) \in R_{F}^{c} \rightarrow x \in A\right)\right) \\
& \text { iff }(\forall x \in X)(y \in F(\{x\}) \rightarrow x \in A)) \\
& \text { iff }\left((\exists x \in X)\left(y \in F(\{x\}) \& x \in A^{c}\right)\right)^{c} \\
& \text { iff } \left.y \in\left(\bigcup_{x \in A^{c}} F(\{x\})\right)\right)^{c} \\
& \text { iff } y \in\left(F\left(\bigcup_{x \in A^{c}}\{x\}\right)\right)^{c}=\left(F\left(A^{c}\right)\right)^{c}=F^{\partial}(A) .
\end{aligned}
$$


(3)

$$
\begin{aligned}
(x, y) \in R_{\langle\langle R\rangle\rangle^{*}} & \text { iff } y \in\langle\langle R\rangle\rangle^{*}(\{x\})^{c} \\
& \text { iff }\left((\exists z \in X)\left((z, y) \in R^{c} \& z \in\{x\}\right)^{c}\right. \\
& \text { iff }(x, y) \in\left(R^{c}\right)^{c}=R .
\end{aligned}
$$

Theorem 3.4. Let $R \in P(X \times Y)$ be a relation.

(1) $[[R]]$ is a sufficiency operator and $\langle\langle R\rangle\rangle$ is a co-sufficiency operator with $\langle\langle R\rangle\rangle(A)=\left([[R]]\left(A^{c}\right)\right)^{c}=[[R]]^{\partial}(A)$ for each $A \in P(X)$.

(2) If $F: P(X) \rightarrow P(Y)$ is a sufficiency operator on $P(X)$, there exists a unique relation $R_{F} \in P(X \times Y)$ such that $\left[\left[R_{F}\right]\right]=F$ and $\left\langle\left\langle R_{F}\right\rangle\right\rangle=F^{\partial}$ where $(x, y) \in R_{F}$ iff $y \in F(\{x\})$.

(3) $R_{[[R]]}=R$.

Proof. (1) We have $\langle\langle R\rangle\rangle(A)=\left([[R]]\left(A^{c}\right)\right)^{c}=[[R]]^{\partial}(A)$ from:

$$
\begin{aligned}
x \in\left([[R]]\left(A^{c}\right)\right)^{c} & \text { iff }\left((\forall y \in X)\left(y \in A^{c} \rightarrow(x, y) \in R\right)\right)^{c} \\
& \text { iff }\left((\forall y \in X)\left((x, y) \in R^{c} \& y \in A^{c}\right)^{c}\right)^{c} \\
& \text { iff }(\exists y \in X)\left((x, y) \in R^{c} \& y \in A^{c}\right) \\
& \text { iff } x \in\langle\langle R\rangle\rangle(A) .
\end{aligned}
$$

(2) Since $F\left(\bigcup_{x \in A}\{x\}\right)=\bigcap_{x \in A} F(\{x\})$, we have

$$
\begin{aligned}
y \in\left[\left[R_{F}\right]\right](A) & \text { iff }(\forall x \in X)\left(x \in A \rightarrow(x, y) \in R_{F}\right) \\
& \text { iff }(\forall x \in X)(x \in A \rightarrow y \in F(\{x\})) \\
& \text { iff } y \in \bigcap_{x \in A} F(\{x\})=F\left(\bigcup_{x \in A}\{x\}\right)=F(A) .
\end{aligned}
$$

$y \in\left\langle\left\langle R_{F}\right\rangle\right\rangle(A)$ iff $(\exists x \in X)\left((x, y) \in R_{F}^{c} \& x \in A^{c}\right)$

iff $(\exists x \in X)\left(y \in F(\{x\})^{c} \& x \in A^{c}\right)$

$$
\begin{aligned}
& \text { iff } y \in \bigcup_{x \in A^{c}} F(\{x\})^{c}=\left(\bigcap_{x \in A^{c}} F(\{x\})\right)^{c} \\
& \text { iff } y \in\left(F\left(\bigcup_{x \in A^{c}}\{x\}\right)\right)^{c}=\left(F\left(A^{c}\right)\right)^{c}=F^{\partial}(A) .
\end{aligned}
$$


(3)

$$
\begin{aligned}
(x, y) \in R_{[[R]]} & \text { iff }(\forall z \in X)(z \in\{x\} \rightarrow(z, y) \in R) \\
& \text { iff }(x, y) \in R .
\end{aligned}
$$

TheOREM 3.5. Let $R \in P(X \times Y)$ be a relation.

(1) $[R]^{*}$ is a sufficiency operator and $\langle R\rangle^{*}$ is a co-sufficiency operator with $[R]^{*}(A)=\left(\langle R\rangle^{*}\left(A^{c}\right)\right)^{c}$.

(2) If $F: P(X) \rightarrow P(Y)$ is a sufficiency operator on $P(X)$, there exists a unique relation $R_{F} \in P(X \times Y)$ such that $\left[R_{F}\right]^{*}=F$ and $\left\langle R_{F}\right\rangle^{*}=F^{\partial}$ where $(x, y) \in R_{F}$ iff $y \in F(\{x\})^{c}$.

(3) $R_{[R]^{*}}=R$.

Proof. (1)

$$
\begin{aligned}
y \in\left(\langle R\rangle^{*}\left(A^{c}\right)\right)^{c} & \text { iff }((\exists x \in X)(x \in A \&(x, y) \in R))^{c} \\
& \text { iff }(\forall x \in X)\left((x, y) \in R \rightarrow x \in A^{c}\right) \\
& \text { iff } y \in[R]^{*}(A) .
\end{aligned}
$$

$(2)$

$$
\begin{aligned}
y \in\left[R_{F}\right]^{*}(A) & \text { iff }(\forall x \in X)\left((x, y) \in R_{F} \rightarrow x \in A^{c}\right) \\
& \text { iff }(\forall x \in X)(x \in A \rightarrow y \in F(\{x\})) \\
& \text { iff } y \in \bigcap_{x \in A} F(\{x\})=F\left(\bigcup_{x \in A}\{x\}\right)=F(A) . \\
y \in\left\langle R_{F}\right\rangle^{*}(A) & \text { iff }(\exists x \in X)\left((x, y) \in R_{F} \& x \in A^{c}\right) \\
& \text { iff }(\exists x \in X)\left(y \in F(\{x\})^{c} \& x \in A^{c}\right) \\
& \text { iff }\left((\forall x \in X)\left(x \in A^{c} \rightarrow y \in F(\{x\})\right)\right)^{c} \\
& \text { iff } y \in\left(\bigcap_{x \in A^{c}}(F(\{x\}))\right)^{c} \\
& \text { iff } y \in\left(F\left(\bigcup_{x \in A^{c}}\{x\}\right)\right)^{c}=\left(F\left(A^{c}\right)\right)^{c} \\
& \text { iff } y \in F^{\partial}(A) .
\end{aligned}
$$


(3)

$$
\begin{aligned}
(x, y) \in R_{[R]^{*}} & \text { iff } y \in[R]^{*}\left(\{x\}^{c}\right)^{c} \\
& \text { iff }\left((\forall z \in X)\left((z, y) \in R \rightarrow z \in\{x\}^{c}\right)\right)^{c} \\
& \operatorname{iff}(x, y) \in R .
\end{aligned}
$$

TheOREM 3.6. Let $R \subset P(X \times Y)$ be a relation.

(1) If $F: P(X) \rightarrow P(Y)$ is a necessity operator on $P(X)$, there exists a unique relation $R_{F} \in P(X \times Y)$ such that $\left[R_{F}\right]=F$ and $\left\langle R_{F}\right\rangle=F^{\partial}$ where $(x, y) \in R_{F}$ iff $y \in F\left(\{x\}^{c}\right)^{c}$.

(2) $R_{[R]}=R$.

Proof. (1)

$$
\begin{aligned}
y \in\left[R_{F}\right](A) & \text { iff }(\forall x \in X)\left((x, y) \in R_{F} \rightarrow x \in A\right) \\
& \text { iff }(\forall x \in X)\left(y \in F\left(\{x\}^{c}\right)^{c} \rightarrow x \in A\right) \\
& \text { iff }(\forall x \in X)\left(x \in A^{c} \rightarrow y \in F\left(\{x\}^{c}\right)\right) \\
& \text { iff } y \in \bigcap_{x \in A^{c}} F\left(\{x\}^{c}\right)=F\left(\bigcap_{x \in A^{c}}\{x\}^{c}\right)=F(A) .
\end{aligned}
$$

$$
y \in\left\langle R_{F}\right\rangle(A) \text { iff }(\exists x \in X)\left((x, y) \in R_{F} \& x \in A\right)
$$$$
\text { iff }(\exists x \in X)\left(y \in F\left(\{x\}^{c}\right)^{c} \& x \in A\right)
$$$$
\text { iff }\left((\forall x \in X)\left(x \in A \rightarrow y \in F\left(\{x\}^{c}\right)\right)\right)^{c}
$$$$
\text { iff } y \in\left(\bigcap_{x \in A} F\left(\{x\}^{c}\right)\right)^{c}=\left(F\left(\bigcap_{x \in A}\{x\}^{c}\right)\right)^{c}
$$

iff $y \in F\left(A^{c}\right)^{c}$ iff $y \in F^{\partial}(A)$.

$(2)$

$$
\begin{aligned}
(x, y) \in R_{[R]} & \text { iff } y \in[R]\left(\{x\}^{c}\right)^{c} \\
& \operatorname{iff}\left((\forall z \in X)\left((z, y) \in R \rightarrow z \in\{x\}^{c}\right)\right)^{c} \\
& \operatorname{iff}(x, y) \in R .
\end{aligned}
$$


Theorem 3.7. Let $R \in P(X \times Y)$ be a relation.

(1) If $F: P(X) \rightarrow P(Y)$ is a co-sufficiency operator on $P(X)$, there exists a unique relation $R_{F} \in P(X \times Y)$ such that $\left\langle\left\langle R_{F}\right\rangle\right\rangle=F$ and $\left[\left[R_{F}\right]\right]=F^{\partial}$ where $(x, y) \in R_{F}$ iff $y \in F\left(\{x\}^{c}\right)^{c}$.

(2) $R_{\left\langle\left\langle R_{F}\right\rangle\right\rangle}=R$.

Proof. (1)

$$
\begin{aligned}
& y \in\left\langle\left\langle R_{F}\right\rangle\right\rangle(A) \text { iff }(\exists x \in X)\left((x, y) \in R_{F}^{c} \& x \in A^{c}\right) \\
& \text { iff }(\exists x \in X)\left(y \in F\left(\{x\}^{c}\right) \& x \in A^{c}\right) \\
& \text { iff } y \in \bigcup_{x \in A^{c}} F\left(\{x\}^{c}\right)=F\left(\bigcap_{x \in A^{c}}\{x\}^{c}\right)=F(A) . \\
& y \in\left[\left[R_{F}\right]\right](A) \text { iff }(\forall x \in X)\left(x \in A \rightarrow(x, y) \in R_{F}\right) \\
& \text { iff }(\forall x \in X)\left(x \in A \rightarrow y \in F\left(\{x\}^{c}\right)^{c}\right) \\
& \qquad \text { iff }\left((\exists x \in X)\left(x \in A \& y \in F\left(\{x\}^{c}\right)\right)\right)^{c} \\
& \qquad \operatorname{iff} y \in\left(\bigcup_{x \in A} F\left(\{x\}^{c}\right)\right)^{c} \\
& \quad \text { iff } y \in\left(F\left(\bigcap_{x \in A}\{x\}^{c}\right)\right)^{c} \\
& \quad \text { iff } y \in F\left(A^{c}\right)^{c}=F^{\partial}(A) .
\end{aligned}
$$

$(2)$

$$
\begin{aligned}
(x, y) \in R_{\langle\langle R\rangle\rangle} & \text { iff } y \in\langle\langle R\rangle\rangle\left(\{x\}^{c}\right)^{c} \\
& \text { iff }\left((\exists z \in X)\left((z, y) \in R^{c} \& z \in\{x\}^{c}\right)\right)^{c} \\
& \text { iff }(x, y) \in R .
\end{aligned}
$$

Theorem 3.8. Let $R \in P(X \times Y)$ be a relation.

(1) If $F: P(X) \rightarrow P(Y)$ is a necessity operator on $P(X)$, there exists a unique relation $R_{F} \in P(X \times Y)$ such that $\left[\left[R_{F}\right]\right]^{*}=F$ and $\left\langle\left\langle R_{F}\right\rangle\right\rangle^{*}=F^{\partial}$ where $(x, y) \in R_{F}$ iff $y \in F\left(\{x\}^{c}\right)$.

(2) $R_{\left[\left[R_{F}\right]\right]^{*}}=R$. 
Proof. (1)

$$
\begin{aligned}
& y \in\left[\left[R_{F}\right]\right]^{*}(A) \text { iff }(\forall x \in X)\left(x \in A^{c} \rightarrow(x, y) \in R_{F}\right) \\
& \text { iff }(\forall x \in X)\left(x \in A^{c} \rightarrow y \in F\left(\{x\}^{c}\right)\right) \\
& \text { iff } y \in \bigcap_{x \in A^{c}} F\left(\{x\}^{c}\right)=F\left(\bigcap_{x \in A^{c}}\{x\}^{c}\right)=F(A) \text {. } \\
& x \in\left\langle\left\langle R_{F}\right\rangle\right\rangle^{*}(A) \text { iff }(\exists x \in X)\left((x, y) \in R_{F}^{c} \& x \in A\right) \\
& \text { iff }(\exists x \in X)\left(y \in F\left(\{x\}^{c}\right)^{c} \& x \in A\right) \\
& \text { iff }\left((\forall x \in X)\left(x \in A \rightarrow y \in F\left(\{x\}^{c}\right)\right)\right)^{c} \\
& \text { iff } y \in\left(\bigcap_{x \in A} F\left(\{x\}^{c}\right)\right)^{c}=\left(F\left(\bigcap_{x \in A}\{x\}^{c}\right)\right)^{c} \\
& \text { iff } y \in F\left(A^{c}\right)^{c} \text { iff } y \in F^{\partial}(A) \text {. }
\end{aligned}
$$

(2)

$$
\begin{aligned}
(x, y) \in R_{\left[\left[R_{F}\right]\right]^{*}} & \text { iff } y \in\left[\left[R_{F}\right]\right]^{*}\left(\{x\}^{c}\right) \\
& \text { iff }(\forall z \in X)(z \in\{x\} \rightarrow(z, y) \in R) \\
& \text { iff }(x, y) \in R .
\end{aligned}
$$

Theorem 3.9. Let $R \in P(X \times Y)$ be a relation.

(1) If $F: P(X) \rightarrow P(Y)$ is a co-sufficiency operator on $P(X)$, there exists a unique relation $R_{F} \in P(X \times Y)$ such that $\left\langle R_{F}\right\rangle^{*}=F$ and $\left[R_{F}\right]^{*}=F^{\partial}$ where $(x, y) \in R_{F}$ iff $y \in F\left(\{x\}^{c}\right)$.

(2) $R_{\left\langle R_{F}\right\rangle^{*}}=R$.

Proof. (1)

$$
\begin{aligned}
y \in\left\langle R_{F}\right\rangle^{*} & \text { iff }(\exists x \in X)\left((x, y) \in R_{F} \& x \in A^{c}\right) \\
& \text { iff }(\exists x \in X)\left(y \in F\left(\{x\}^{c}\right) \& x \in A^{c}\right) \\
& \text { iff } y \in \bigcup_{x \in A^{c}} F\left(\{x\}^{c}\right)=F\left(\bigcap_{x \in A^{c}}\{x\}^{c}\right)=F(A) .
\end{aligned}
$$




$$
\begin{aligned}
y \in\left[R_{F}\right]^{*}(A) & \text { iff }(\forall x \in X)\left(x \in(x, y) \in R_{F} \rightarrow x \in A^{c}\right) \\
& \text { iff }\left((\exists x \in X)\left(x \in A \&(x, y) \in R_{F}\right)\right)^{c} \\
& \text { iff }\left((\exists x \in X)\left(x \in A \& y \in F\left(\{x\}^{c}\right)\right)\right)^{c} \\
& \text { iff } y \in\left(\bigcup_{x \in A} F\left(\{x\}^{c}\right)\right)^{c} \\
& \text { iff } y \in\left(F\left(\bigcap_{x \in A}\{x\}^{c}\right)\right)^{c} \\
& \text { iff } y \in F\left(A^{c}\right)^{c}=F^{\partial}(A) .
\end{aligned}
$$

(2)

$$
\begin{aligned}
(x, y) \in R_{\left\langle R_{F}\right\rangle^{*}} & \text { iff } y \in\left\langle R_{F}\right\rangle^{*}\left(\{x\}^{c}\right) \\
& \text { iff }\left((\exists z \in X)\left((z, y) \in R^{c} \& z \in\{x\}\right)\right)^{c} \\
& \text { iff }(x, y) \in R .
\end{aligned}
$$

Example 3.10. Let $X=\{a, b, c\}$ and $Y=\{x, y, z\}$ be a set. Define $F, G: P(X) \rightarrow P(Y)$ as

$$
\begin{gathered}
F(\{a\})=\emptyset, F(\{b\})=\{x\}, F(\{c\})=\{y, z\}, \\
G(\{a\})=X, G(\{b\})=\{x, y\}, G(\{c\})=\{y, z\}, \\
H(\{b, c\})=\{x\}, H(\{c, a\})=\{x, y\}, H(\{a, b\})=\{z\} .
\end{gathered}
$$

(1) If $F$ is a modal operator, then, by Theorem 2.3,

$$
F(A)= \begin{cases}\emptyset, & \text { if } A \in\{\emptyset,\{a\}\}, \\ \{x\}, & \text { if } A \in\{\{b\},\{a, b\}\}, \\ \{y, z\}, & \text { if } A \in\{\{c\},\{a, c\}\}, \\ Y, & \text { if } A \in\{\{b, c\}, X\} .\end{cases}
$$


Since $(x, y) \in R_{F}$ iff $y \in F(\{x\})$, we obtain:

$$
R_{F}=\{(b, x),(c, y),(c, z)\},\left\langle R_{F}\right\rangle=F,\left[R_{F}\right]=F^{\partial} .
$$

(2) If $F$ is a modal operator, then, by Theorem 3.3, we obtain $F$ as same in (1). Since $(x, y) \in R_{F}$ iff $y \in F(\{x\})^{c}$, we obtain:

$$
\begin{gathered}
R_{F}=\{(a, x),(a, y),(a, z),(b, y),(b, z),(c, x)\}, \\
\left\langle\left\langle R_{F}\right\rangle\right\rangle^{*}=F,\left[\left[R_{F}\right]\right]^{*}=F^{\partial} .
\end{gathered}
$$

(3) If $G$ is a sufficiency operator, then, by Theorem 3.4,

$$
G(A)= \begin{cases}Y, & \text { if } A \in\{\emptyset,\{a\}\}, \\ \{x, y\}, & \text { if } A \in\{\{b\},\{a, b\}\}, \\ \{y, z\}, & \text { if } A \in\{\{c\},\{a, c\}\}, \\ \{y\}, & \text { if } A \in\{\{b, c\}, X\} .\end{cases}
$$

Since $(x, y) \in R_{G}$ iff $y \in G(\{x\})$, we obtain:

$$
\begin{gathered}
R_{G}=\{(a, x),(a, y),(a, z),(b, x),(b, y),(c, y),(c, z)\} \\
{\left[\left[R_{G}\right]\right]=G,\left\langle\left\langle R_{G}\right\rangle\right\rangle=G^{\partial} .}
\end{gathered}
$$

(4) If $G$ is a sufficiency operator, then, by Theorem 3.5, we obtain $G$ as same in (3). Since $(x, y) \in R_{G}$ iff $y \in G(\{x\})^{c}$, we obtain:

$$
R_{G}=\{(b, z),(c, x)\},\left[R_{G}\right]^{*}=G,\left\langle R_{G}\right\rangle^{*}=G^{\partial} .
$$

(5) If $H$ is a necessity operator, then, by Theorem 3.6,

$$
H(A)= \begin{cases}\emptyset, & \text { if } A \in\{\emptyset,\{a\},\{b\}\}, \\ \{x\}, & \text { if } A \in\{\{c\},\{b, c\}\}, \\ \{x, y\}, & \text { if } A=\{a, c\}, \\ \{z\}, & \text { if } A=\{a, b\}, \\ Y, & \text { if } A=X .\end{cases}
$$


Since $(x, y) \in R_{H}$ iff $y \in H\left(\{x\}^{c}\right)^{c}$, we obtain:

$$
R_{H}=\{(a, y),(a, z),(b, z),(c, x),(c, y)\},\left[R_{H}\right]=H,\left\langle R_{H}\right\rangle=H^{\partial} .
$$

(6) If $H$ is a necessity operator, then, by Theorem 3.8, we obtain $H$ as same in (5). Since $(x, y) \in R_{H}$ iff $y \in H\left(\{x\}^{c}\right)$, we obtain:

$$
R_{H}=\{(a, x),(b, x),(b, y),(c, z)\},\left[\left[R_{H}\right]\right]^{*}=H,\left\langle\left\langle R_{H}\right\rangle\right\rangle^{*}=H^{\partial} .
$$

(7) If $H$ is a co-sufficiency operator, then, by Theorem 3.7, we have:

$$
H(A)= \begin{cases}\emptyset, & \text { if } A=X, \\ \{x, y\}, & \text { if } A \in\{\{c\},\{a, c\}\}, \\ \{x, z\}, & \text { if } A=\{b\}, \\ \{z\}, & \text { if } A=\{a, b\}, \\ \{x\}, & \text { if } A=\{b, c\}, \\ Y, & \text { if } A \in\{\emptyset,\{a\}\} .\end{cases}
$$

Since $(x, y) \in R_{H}$ iff $y \in H\left(\{x\}^{c}\right)^{c}$, we obtain:

$$
R_{H}=\{(a, y),(a, z),(b, z),(c, x),(c, y)\},\left\langle\left\langle R_{H}\right\rangle\right\rangle=H,\left[\left[R_{H}\right]\right]=H^{\partial} .
$$

(8) If $H$ is a co-sufficiency operator, then, by Theorem 3.9, we obtain $H$ as same in (7). Since $(x, y) \in R_{H}$ iff $y \in H\left(\{x\}^{c}\right)$, we obtain:

$$
R_{H}=\{(a, x),(b, x),(b, y),(c, z)\},\left\langle R_{H}\right\rangle^{*}=H,\left[R_{H}\right]^{*}=H^{\partial} .
$$

\section{References}

[1] R. Bělohlávek, Lattices of fixed points of Galois connections, Math. Logic Quart. 47 (2001), 111-116.

[2] I. Düntsch, Ewa. Orlowska, Boolean algebras arising from information systems, Ann. Pure Appl. Logic 127 (2004), 77-98.

[3] J. Järvinen, M. Kondo, J. Kortelainen, Logics from Galois connections, Internat. J. Approx. Reason. 49 (2008), 595-606.

[4] Ewa. Orlowska, I. Rewitzky, Algebras for Galois-style connections and their discrete duality, Fuzzy Sets and Systems 161 (2010), 1325-1342.

[5] Z. Pawlak, Rough sets, Int.J .Comput. Sci. Math. 11 (1982), 341-356. 
[6] G. Qi, W. Liu, Rough operations on Boolean algebras, Inform. Sci. 173 (2005), 49-63.

[7] R. Wille, Restructuring lattice theory; an approach based on hierarchies of concept, in: 1. Rival(Ed.), Ordered Sets, Reidel, Dordrecht, Boston (1982).

[8] W. Yao, L.X. Lu, Fuzzy Galois connections on fuzzy posets, Math. Logic Quart. 55 (2009), 105-112.

[9] Y.Y. Yao, Two Views of the Theory of Rough Swts in Finite Universes, Internat. J. Approx. Reason. 15 (1996), 291-317.

Department of Mathematics

Natural Science

Gangneung-Wonju National University

Gangneung, 210-702, Korea

E-mail: yck@gwnu.ac.kr 